\title{
Failure of Immature Lungs to Produce Foam and Retain Air at Birth
}

\author{
EMILE M. SCARPELLI, ${ }^{(23)}$ BELLA C. CLUTARIO, AND DIANE TRAVER \\ Pediatric Pulmonary Division, Albert Einstein College of Medicine, Bronx, New York, USA
}

\section{Summary}

Immature and mature rabbit neonates were observed for production of intrapulmonary foam during the first 5-10 min of extrauterine life. Viability at birth of littermates was determined. In addition, lungs from neonates that had breathed 3-5 min after birth and from fetuses that had not breathed were studied during manual inflation to maximal volume and deflation to zero transpulmonary pressure (minimal volume). Lung wet/dry weights and amniotic fluid lecithin-to-sphingomyelin ratios were determined. Compared with mature subjects, body weights and lecithin-tosphingomyelin ratios were significantly lower in the immature group, and total water content was significantly higher. No immature neonate lived to $3 \mathrm{hr}$, whereas all mature neonates were robust, active, and normal. Both spontaneous breathing up to 10 min and manual inflation-deflation were associated with intrapulmonary foam in each mature lung, but with little or no foam in immature lungs. The following were characteristic of immature lungs: 1) Airways and terminal lung units (TLU) were generally liquid-filled at zero transpulmonary pressure. 2) When lungs were inflated to maximal volume, all TLU appeared aerated. 3) However, during deflation liquid refilled most TLU and airways and $>95 \%$ of the air previously introduced was returned to the syringe. 4) Air trapped in TLU at end deflation was not due to airway collapse, but to relatively early refilling of more proximal adjacent airways which thereby prevented air from leaving more peripheral TLU. Similar studies of mature lungs confirmed our previous report (Pediatr. Res., 12: 1070 (1978)) that intrapulmonary foam is produced at the onset of breathing at birth and, also, during initial manual inflations of the liquid-filled fetal lung. Thus, the striking difference between the mature and immature lungs is the inability of the latter to produce foam. This is related to immaturity of the surfactant system, inability to establish residual volume at the onset of breathing, and retention of liquid in potential air spaces.

\section{Speculation}

Immaturity of the surfactant system inhibits the production of intrapulmonary foam at the onset of air-breathing at birth. This deficiency, which is characteristic of immature lungs, retards residual volume formation and fetal pulmonary fluid absorption. Therefore, pulmonary blood flow, gas exchange, and alveolar lining formation are compromised.

It has been reported that immediate establishment of residual gas volume in the lungs at the onset of breathing at birth is due, in large part, to the production of intrapulmonary foam as air first enters the previously liquid-filled lungs (15). This process was shown to be a normal adaptational phenomenon for mature lungs and, on theoretical grounds, to be integral to the known cardiopulmonary adjustments that insure immediate and sustained survival outside the uterus, viz., decrease of pulmonary vascular resistance, increase of pulmonary blood flow, establishment of functional residual capacity (FRC) and formation of the alveolar lining layer (ALL) (15). In the present studies, immature and mature lungs of rabbits were compared with regard to production of neonatal intrapulmonary foam and to survival at birth. These studies included both gross and microscopic inspection of lungs from neonates up to $10 \mathrm{~min}$ after onset of breathing and from fetuses in whom the first inflations and deflations with air were administered from a calibrated syringe. It has been shown that nonviable immature neonates, in contrast with the viable mature animals, were neither capable of producing foam nor able to sustain a residual pulmonary gas volume. The possibility is suggested that the former deficiency, which results from immaturity of the pulmonary surfactant system, is related causally to the latter.

\section{METHODS}

Twenty-four pregnant New Zealand White rabbits were prepared as described previously (15) and fetuses were delivered through uterotomy at either 26-28 days of gestation (17 rabbits, immature group) or $>28$ days of gestation ( 7 rabbits, mature group).

Neonates from the mature litters and from six of the immature litters were handled as follows: subgroup 1: in order to determine extrauterine viability, 2-3 neonates from each litter were permitted to breathe spontaneously under a radiant heater and observed for spontaneous activity, "cry," color, breathing movements, and survival time. The skin was gently stroked regularly during the first $10 \mathrm{~min}$ to stimulate breathing movements. Subgroup 2: an additional 1-2 neonates from each litter were handled the same way, but killed by ip pentobarbital overdose 5-10 min after birth. The chests were opened by sternotomy and lungs examined immediately for the presence of airway foam as described previously (15). Subgroup 3: 1-2 additional neonates from each immature litter and one neonate from three mature litters were killed 3-5 min after the onset of breathing and the lungs examined by stereomicroscopy (20) according to the following protocol: the anterior thorax was excised and the lungs were scanned, in situ, through the microscope as a cannula was inserted into the trachea by a second experimenter. The lungs of immature neonates were inflated with a volume of air equivalent to estimated FRC from a calibrated syringe: FRC was estimated as body weight $(\mathrm{g}) \times 30$ $\mu \mathrm{l}$, according to data obtained by the authors in previous experiments in which fetal pulmonary fluid (FPF) volume was measured in lamb fetuses in vivo (17). FPF volume is about $30 \mathrm{ml} / \mathrm{kg}$, which approximates normal FRC $(14,17)$. This procedure simulated the first breath of normal human infants as reported by Karlberg et al. (11); it could be applied to immature neonates because their lungs were generally liquid-filled. Mature lungs, however, were well-aerated so that end-inflation was determined as the point at which all visible TLU (21) were inflated. The volume required for mature lungs ranged from 0.3-0.5 FRC. Lungs were then deflated to minimal volume, zero transpulmonary pressure, and inflationdeflation cycles were repeated up to 10 times. Subgroup 4: whole lungs of immature neonates that had died after 3-5 min of spontaneous air-breathing were weighed "wet" and again after drying in an oven to determine percent of lung water (1); 1-2 
neonates from each mature litter, randomly selected, were killed after 3-5 min of air-breathing and wet/dry weights were determined for comparison. Dry weight was obtained when lung weight had become constant.

Thirty-three fetuses from the remaining eleven immature litters were killed in utero, anterior chest wall excised, and lung immediately scanned by stereomicroscopy during initial air inflations and deflations according to the protocol previously described for subgroup 3 which is the same protocol used in our previous study of mature fetal lungs (15). Amniotic fluid was obtained before delivery of fetuses and analyzed for lecithin-to-sphingomyelin ratio by standard techniques reported previously (18), but without acetone precipitation. These were compared with amniotic fluid obtained from mature pregnancies.

\section{RESULTS}

Lecithin-to-sphingomyelin ratio of the immature group was $0.01-0.6$, whereas the ratio for the mature group was 1.85-2.6. Data from the neonatal studies are presented in Table 1 and outlined in this paragraph. Body weight of the immature rabbits was significantly lower than that of the mature rabbits $(P<0.01$, unpaired $t$ test). Percent lung water of immature neonates that had breathed spontaneously 3-5 min was significantly greater than that of mature neonates killed 3-5 min after the onset of breathing $(P<0.01)$ (subgroup 4 ). Immature neonates made frequent breathing movements with tactile stimulation, but none lived longer than $3 \mathrm{hr}$ after birth, whereas all mature neonates that were observed were robust and normal by all criteria (subgroup 1) Except for one immature neonate in which few bubbles were present in the trachea, none of the lungs of the immature rabbits examined 5-10 min after the onset of breathing contained foam in the large airways; conversely, foam was seen in large airways (trachea or $1^{\circ}, 2^{\circ}, 3^{\circ}$ bronchi) of each mature neonate (subgroup 2).

Stereomicroscopic examination of immature neonatal lungs (air breathing 3-5 min; subgroup 3 ) revealed the following: 1) airways and TLU were generally filled with liquid; 2 ) few TLU contained air; 3 ) when lungs were inflated, all visible TLU appeared aerated (Fig. 1). This appearance was not readily distinguishable from that of inflated mature lungs (see later in this paper and ref. 15). 4) However, during deflation, liquid, which had been moved to the periphery of TLU during inflation, refilled most TLU and airways. Virtually all the air (>95\%) introduced into the lungs during inflation was returned during deflation. Air entrapment during deflation was due to the different rates at which adjacent airways and TLU became refilled with liquid. Thus, if one unit had refilled with liquid before the adjacent unit had emptied of air, the liquid from the first unit - once it had entered the common airway-prevented further emptying of air from the second unit. This entrapped air (as in Fig. 2) could be moved with a micromanipulator by the experimenter and, with the micromanipulator, could be transferred easily from one unit to another. The configuration was either spherical or cylindrical. The latter forms tended to enlarge during subsequent inflations to maximal volume and,

Table 1. Comparison of mature and immature neonates ${ }^{1,2}$

\begin{tabular}{lll}
\hline & \multicolumn{1}{c}{$26-28$ days } & \multicolumn{1}{c}{$>28$ days } \\
\hline No. Litters & 6 & 7 \\
Body wt $(\mathrm{g})$ & $17.6(9.5-28.6)$ & $34.6(26.1-46.4)$ \\
& {$[\mathrm{n}=37]$} & {$[\mathrm{n}=39]$} \\
NB status & Died $<3 \mathrm{hr}$ & Robust, active \\
$\quad$ (subgroup 1) & $\cdot[\mathrm{n}=14]$ & {$[\mathrm{n}=16]$} \\
Foam in large airways & None (7); trace (1) & All \\
$\quad$ (subgroup 2) & {$[\mathrm{n}=8]$} & {$[\mathrm{n}=12]$} \\
Lung water (\%) & $87.3 \pm 1.3$ & $81.4 \pm 2.3$ \\
$\quad$ (subgroup 4) & {$[\mathrm{n}=16]$} & {$[\mathrm{n}=8]$} \\
\hline
\end{tabular}

${ }^{1} \mathrm{n}=$ number of observations.

${ }^{2}$ Range of body wt and mean \pm SD of \% lung water are given.

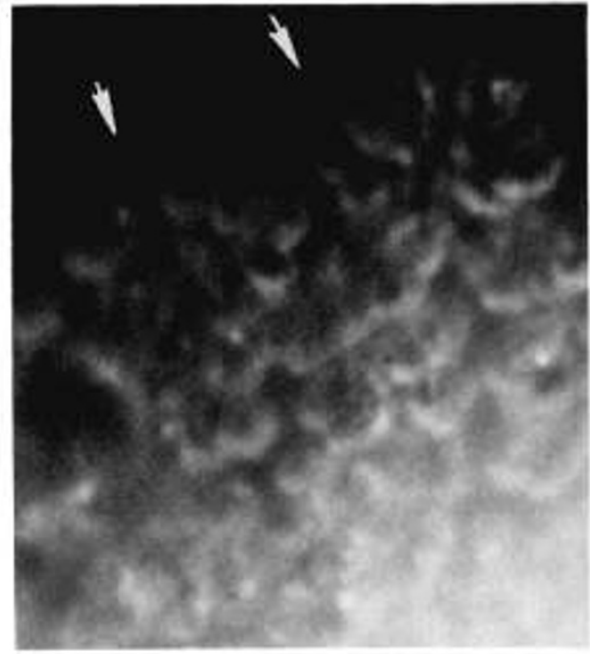

Fig. 1. Lung surface showing aeration of $12 \mathrm{LU}$ atter intlation to maximal volume. Immature rabbit lung, 27.5 days of gestation. Arrows point to edge of pulmonary segment. $\times 45$.

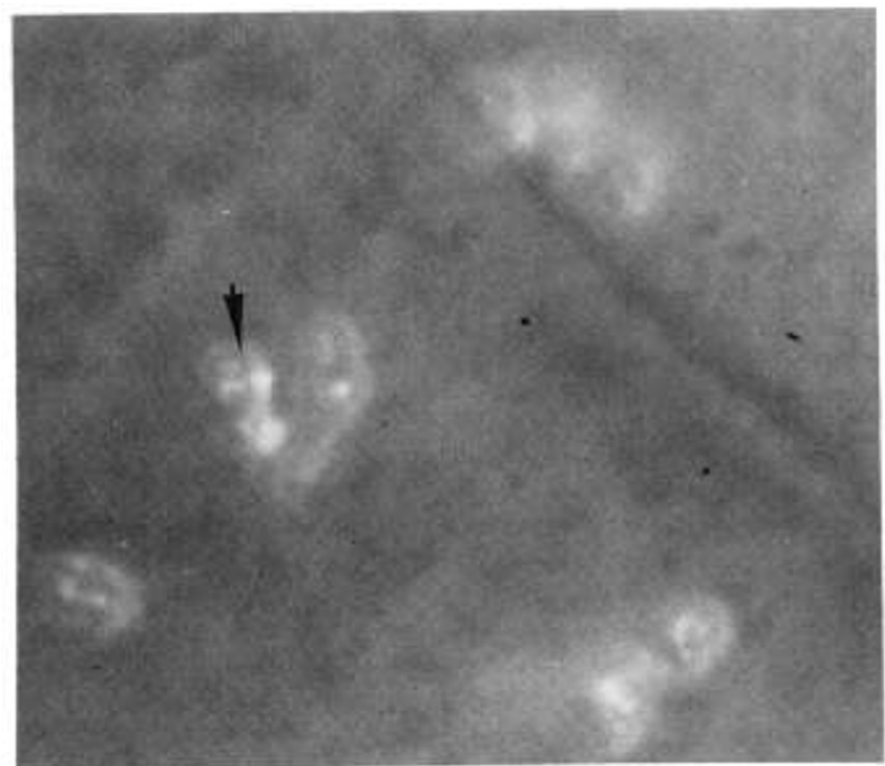

Fig. 2. Surface of adjoining segments of immature (27.5 days of gestation) rabbit lung at end-deflation, zero transpulmonary pressure, which followed inflation to maximal volume. Most of the lung remains liquidfilled; trapped gas is retained in few areas (e.g., arrow). $\times 94$.

thus, probably were not bound by films. Thus, entrapment was not due to foam formation.

Stereomicroscopic examination of immature fetal lungs corroborated this: initial aeration was seen as air entered first the larger then terminal airways and as TLU expanded (Fig. 3). With inflation, all visible TLU became air-filled (Fig. 1). During deflation, liquid, which had been displaced peripherally during inflation, refilled TLU and airways; the air-liquid interface moved from periphery of TLU to the larger airways as deflation proceeded. Virtually all air $(>95 \%)$ was returned to the syringe. At end-deflation, few areas of the lung had retained air (Fig. 2). Air entrapment was as described for the immature neonatal lungs.

With regard to the inflation of immature lungs, it should be noted that no leaks were produced at maximal volume and that the extent of aeration of TLU at maximal volume was comparable, by gross and microscopic inspection, to that of mature lungs inflated with equivalent volumes (15), so that opening pressure had been exceeded. It is during deflation that the absence of foam is best appreciated in immature lungs. 


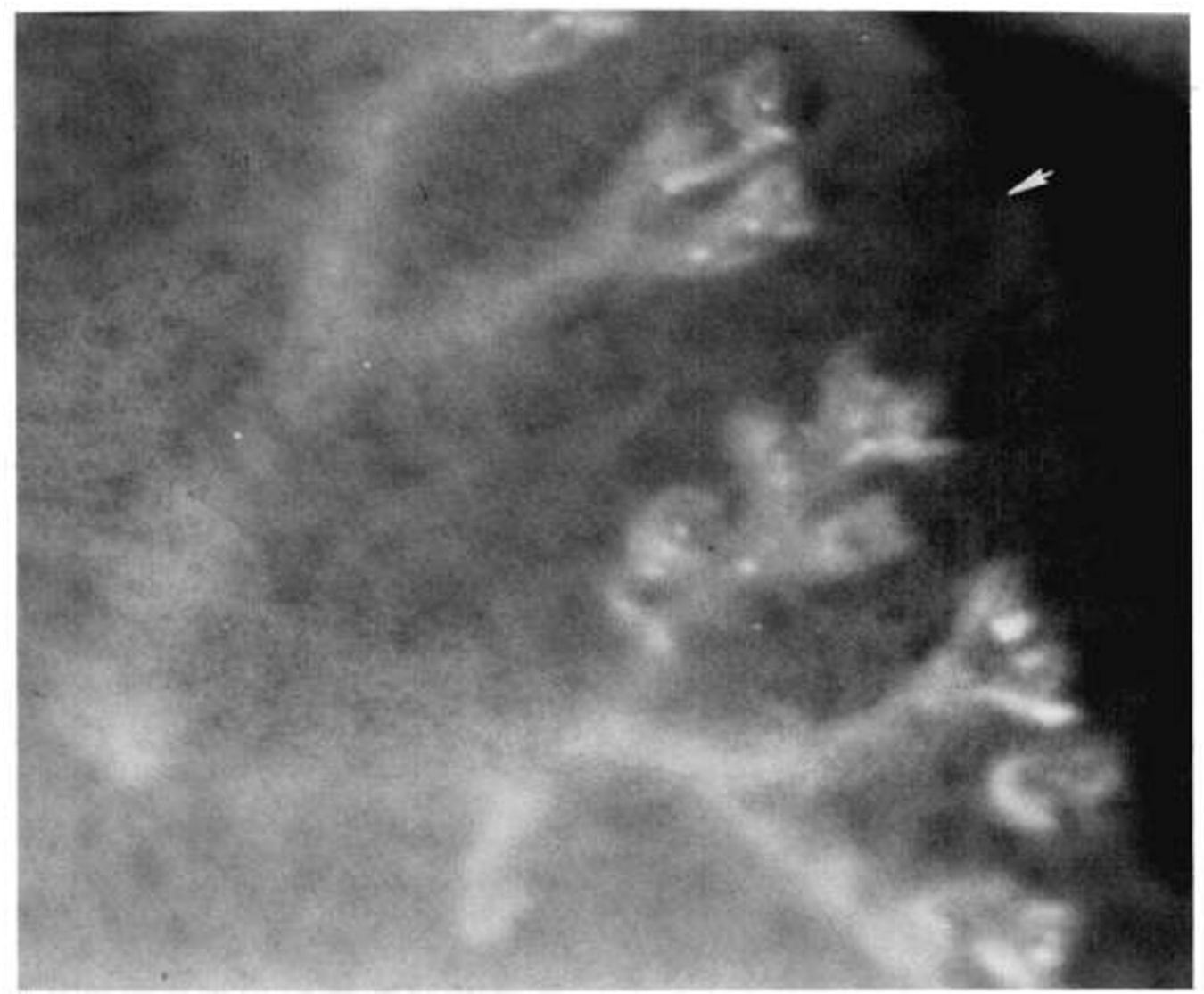

Fig. 3. Early aeration of immature (27.5 days of gestation) rabbit lung. Most of the lung is still liquid-filled. Air has entered major airways, terminal airways, and TLU shown. As inflation was continued beyond this point, additional airways and units were recruited until virtually all units were aerated at maximal volume as indicated in Figure 1. Arrow points to edge of pulmonary segment. $\times 94$.

Stereomicroscopic examination of mature neonatal lungs was consistent with previous studies (15): lungs were generally wellaerated. A superficial incision of the surface with scalpel yielded foam. During inflation of intact lungs to maximal volume, bubbles moved into TLU where they were retained at end-deflation.

\section{DISCUSSION}

Mature subjects of the present studies were robust and active at birth. Their lungs contained significantly less total liquid than those of immature subjects after 3-5 min of spontaneous airbreathing, their amniotic fluid lecithin-to-sphingomyelin ratio was greater than immature subjects' and indicative of pulmonary maturity, and intrapulmonary foam was present in each lung examined up to 10 min after onset of breathing and in all lungs inflated manually to maximal volume. The latter is consistent with the previous study of mature lungs by the authors (15).

In contrast, immature rabbits died within $3 \mathrm{hr}$. Their lecithinto-sphingomyelin ratio was $\leqq 0.6$ and, thus, indicative of immaturity of the surfactant system. Indeed, it has been shown that immature mammalian lungs have low phospholipid surfactant content and, therefore, low quantities in $\operatorname{FPF}(6,7)$. The lecithin content of rabbit FPF does not begin to increase until after 28 days of gestation $(6,7)$, the same time that stable extracts and stable volume-pressure curves are first obtained from the lungs $(10,12)$. Presumably, also, the immature lung is not able to mobilize surfactant secretion at birth (7), which may further compromise neonatal lung stability. These measurements of wet/ dry weights also show that immature lungs contain significantly high quantities of liquid after 3-5 minutes of air-breathing. Although this liquid is not exclusively FPF (1), this finding is in accord with previous demonstrations of slow absorption of FPF from immature lungs after birth (9) and with the present observations of retained liquid in potential air spaces. Although these data suggest very rapid clearance of lung liquid in mature lungs, the authors have no information about comparative rates of clearance because FPF volumes were not measured and weights were not obtained before or after 3-5 min. Finally, these studies demonstrate that little or no intrapulmonary foam is produced either during spontaneous air-breathing at birth or during manual inflation of the lungs in situ. The immature lung is virtually airless in the absence of distending transpulmonary pressures, i.e., it cannot sustain adequate residual volume and is is unstable. "Instability" for these lungs is not characterized by the traditional "collapse" of previously air-filled TLU, but rather by refilling of TLU with liquid during and at end-deflation. The striking difference between these and mature lungs is their incapacity to produce significant quantities of intrapulmonary foam.

The general concept that emerges from these observations is that with maturation of the surfactant system, the capacity to produce foam at the onset of breathing is established. It has been suggested (15) that among the physicochemical factors (3) that influence foam production in situ, the content of surfactants in FPF and, perhaps, enhanced secretion at birth (7) are of central importance. In fact, intrapulmonary foam production may be compared with the process of foam fractionation in vitro (16) which, for example, has been used to recover phospholipid surfactants in foam films produced from mature FPF (5). It has also been shown that FPF surfactants are incorporated, apparently preferentially, into bubble films when foam is produced in mature lungs (15). From these and the present studies, it is apparent that surfactant deficiency or immaturity is associated with poor foam production at the onset of air-breathing. Therefore, it seems reasonable to conclude that prenatal maturation of the surfactant system establishes the capacity to produce foam at the onset of breathing. Whether or not foam production is essential for residual volume formation during the first minutes of extrauterine life may still be questioned. The possibility may be raised that lack of foam 
may result from, rather than cause, inability to establish residual volume by other mechanisms. These experiments show that intrapulmonary foam production and immediate establishment of residual volume are coincident in mature lungs (15) and that both these adaptational phenomena are compromised in immature lungs.

In a previous report (15), the possible central role of early neonatal foam in normal cardiopulmonary adaptations at birth was emphasized including rapid oxygenation of blood, maintenance of airway and TLU distention at the start of breathing, and formation of the ALL of the neonatal air-lung. It was suggested that, from this perspective, the "foam state"-in which the lung is a three phase system of air, liquid, and foam-is a short-lived but crucial normal adaptational phenomenon. The present studies indicate that this capacity is compromised by pulmonary immaturity. Further, they suggest an additional function of foam, i.e., in FPF absorption at birth. Although the mechanism underlying absorption is not known, it has been shown that bubbles in TLU produce and sustain immediate distention of these units (15). This, in turn, may be related to the reported (4) increase of equivalent pore size of epithelial cells, which may promote movement of FPF out of potential air spaces (4). Immature lungs that lack foam tend to retain intraluminal liquid.

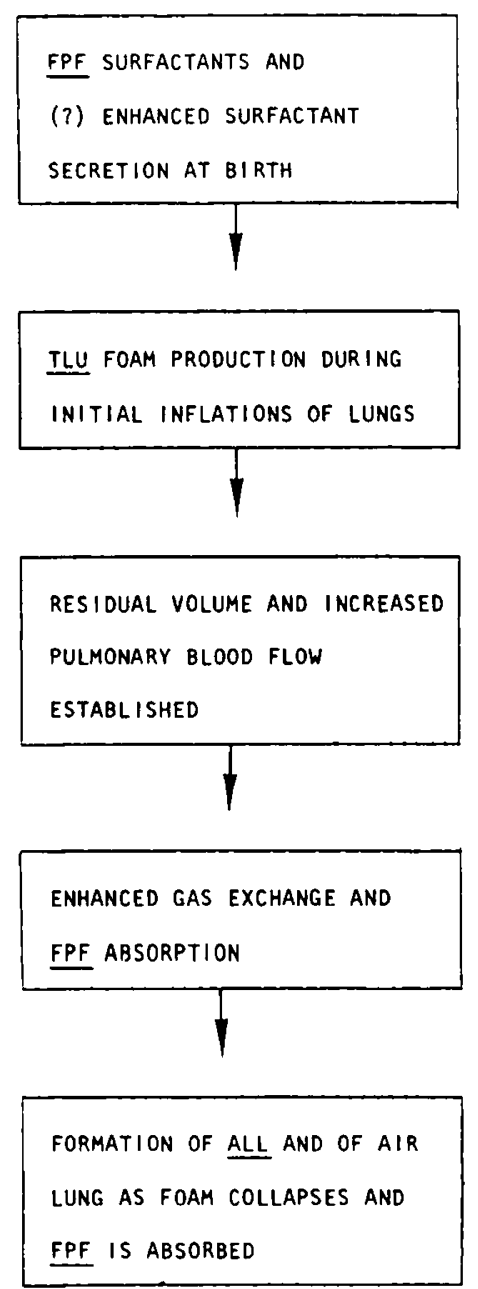

Fig. 4. Resume of proposed role of intrapulmonary foam during the first minutes of air breathing which leads ultimately to establishment of the normal air lung. Capacity to produce intrapulmonary foam depends on prior maturation of the surfactant system. Foam formation is the basis for immediate establishment of residual volume and, perhaps, for ultimate formation of the alveolar lining layer. Compared with mature lungs, immature lungs lack this capacity.
Morphologic characteristics of immature lungs, as determined by stereomicroscopy, sustain these concepts. Although inflation of these lungs to maximal volume produces apparent aeration of all TLU, the liquid displaced to the priphery during inflation refills most of the lumina on deflation. The liquid moves from periphery of TLU into the airways. Presumably, this process would be augmented in vivo by the high positive pleural pressure produced during expiration at the onset of breathing (11). The refilling phenomenon is also seen in some TLU of mature lungs (15). However, whereas it is the exception in the latter, it is a characteristic feature of immature lungs. Refilling is consistent with reported functional characteristics of immature lungs at birth, viz., slow absorption of FPF (9), small FRC (2), and systemic hypoxemia to which intrapulmonary shunts are major contributors (13, 19).

Some air trapping was observed in immature TLU during deflation. Areas of entrapment were often distended further during subsequent inflations and remained distended between inflations at zero transpulmonary pressure. Such entrapment was not the result of proximal airway collapse, but of liquid refilling airways from adjacent TLU. This trapped gas could be a primary site for gas exchange in immature lungs at the onset of breathing. However, progressive distention might also lead to tissue damage and rupture.

A resume of the foam lung, including its suggested significance during the first minutes of extrauterine life, is given in Figure 4.

\section{CONCLUSION}

Maturation of the lung surfactant system establishes the milieu for intrapulmonary foam formation at the onset of air-breathing at birth. This is a normal characteristic of mature lungs, but is impaired or absent in immature lungs. The expected consequences are retardation of residual volume formation and retention of FPF in potential air spaces. This, in turn, compromises pulmonary blood flow, pulmonary gas exchange, and ultimate formation of the ALL. Although the foam state is a relatively short transient in the period after the onset of breathing, it is probably integral to the essential cardiopulmonary adjustments that characterize early neonatal transition from the uterine environment.

\section{REFERENCES AND NOTES}

I. Adams, F. H., Yanagisawa, M., Kuzela, D., and Martinek, H.: The disappearance of fetal lung fluid following birth. J. Pediatr. 78 : 837 (1971)

2. Auld, P. A. M.: Pulmonary physiology of the newborn infant. In: E. M. Scarpelli and P. A. M. Auld: Pulmonary Physiology of the Fetus, Newborn and Child, p. 144, (Lea and Febiger, Philadelphia, 1975).

3. Bikerman, J. J.: Foams, Chapt. 2, (Springer-Verlag, New York, 1973)

4. Egan, E. A., Olver, R. E., and Strang, L. B.: Changes in non-electrolyte perme ability of alveoli and the absorption of lung tiquid at the start of breathing in the lamb. J. Physiol. (London), 244: 161 (1975).

5. Enhorning, G., Fujiwara, T., and Adams, F. H.: Surface-active material in fetal lung fluid concentrated by foaming. Tohoku J. Exptl. Med., 84: 250 (1964).

6. Gluck, L., Motoyama, E. K., Smits, H. L., and Kulovich, M. V.: The biochemical development of surface activity in mammalian lung. I. The surface-active phospholipids; the separation and distribution of surface-active lecithin in the lung of the developing rabbit fetus. Pediatr. Res., 1: 237 (1967).

7. Gluck, L., Sribney, M., and Kulovich, M. V.: The biochemical development of surface activity in mammalian lung, II. The biosynthesis of phospholipids in the lung of the developing rabbit fetus and newborn. Pediatr. Res., 1: 247 (1967).

8. Hislop, A., and Reid, L.: Development of the acinus in the human lung. Thorax, 29: 90 (1974)

9. Humphreys, P. W., Normand, I. C. S., Reynolds, E. O. R., and Strang, L. B.: Pulmonary lymph flow and the uptake of liquid from the lungs of the lamb at the start of breathing. J. Physiol. (London), 193: 1 (1967).

10. Humphreys, P. W., and Strang. L. B.: Effects of gestation and prenatal asphyxia on pulmonary surface properties of the foetal rabbit. J. Physiol. (London), 192: 53 (1967).

11. Karlberg, P.. Cherry, R. B., Escardo, F. E., and Koch, G.: Respiratory studies in newborn infants. II. Pulmonary ventilation and mechanics of breathing in the first minutes of life, including the onset of respiration. Acta Paediatr. (Scand.), 51: 121 (1962).

12. Kotas, R. V., and Avery, M. E.: Accelerated appearance of pulmonary surfactant in the fetal rabbit. J. Appl. Physiol., 30: 358 (1971).

13. Krauss, A. N., and Auld, P. A. M.: Ventilation-perfusion abnormalities in the 
premature infant. Triple gradient. Pediatr. Res., 3: 255 (1969).

14. Scarpelli, E. M.: Fetal pulmonary fluid. Rev. Perinat. Med., 1: 49 (1976)

15. Scarpelli, E. M.: Intrapulmonary foam at birth: an adaptational phenomenon. Pediatr. Res., 12: 1070 (1978).

16. Scarpelli, E. M.: The Surfactant System of the Lung, p. 58, (Lea and Febiger, Philadelphia, 1968)

17. Scarpelli, E. M., Condorelli, S., and Cosmi, E. V.: Lamb fetal pulmonary fluid. I. Validation and significance of method for determination of volume and volume change. Pediatr. Res., 9: 190 (1975)

18. Schulman, J. D., Queenan, J. T., Scarpelli, E. M., Church, E., and Auld, P. A. M.: Lecithin-sphinogomyelin ratios in amniotic fluid and their relation to neonatal condition and gestational age. Obstet. Gynecol., 40: 697 (1972)

19. Thibeault, D. W., Wong, M. M., and Auld. P. A. M.: Alveolar-arterial O.2 and $\mathrm{CO}_{2}$ differences and their relation to lung volume in the newborn. Pediatrics, 4l: 574, (1968).

Copyright $(\mathcal{C} 1979$ International Pediatric Research Foundation, Inc $0031-3998 / 79 / 1311-1285 \$ 02.00 / 0$
20. Wild Heerbrugg stereo microscope, model M5, Wild Corp., Switzerland.

21. The term "terminal lung unit(s)" (TLU) as used in this report includes both conducting structures, which we refer to as "airways," and terminal limiting structures, which we refer to as "periphery." Airways would be analogous to "transitional ducts" and "saccules"; and periphery would be analogous to "terminal saccules" as discussed by Hislop and Reid (8). The generally accepted nomenclature (8) has not been used because microscopic resolution in our preparations did not permit precise anatomic definition at the cellular level. 22. This research was supported by a grant from the National Heart, Lung and Blood Institute, HL 16137, National Institutes of Health.

23. Requests for reprints should be addressed to: Dr. Emile M. Scarpelli, Pediatric Pulmonary Division, Albert Einstein College of Medicine, 1300 Morris Park Avenue, Bronx, New York 10461, USA.

24. Received for publication November 15,1978

25. Accepted for publication July 5, 1979. 